\title{
Oral squamous cell carcinoma; Long term prognosis in a low- middle income country
}

Rahim Dhanani, Hamdan Ahmed Pasha, Fahad Rind, Kulsoom Ghias, Mumtaz Jamshed Khan

Section of Otolaryngology/Head and neck surgery, Department of Surgery, Aga Khan University Hospital, Karachi, Pakistan

Background: Clinical outcome of oral squamous cell carcinoma patients has remained unchanged despite advances in treatment modalities. In the absence of a national cancer registry in our country, only institutional based data is available. We review our patients of oral carcinoma to identify factors influencing recurrence and long term survival.

Objective: To identify factors influencing recurrence and long term survival of patients with oral squamous cell carcinoma.

Methods: All patients operated for oral cavity squamous cell carcinoma between 2009 and 2012 were reviewed. Patient data was abstracted from the charts and institutional database. Survival analysis was performed with Kaplan Meier methods, while differences in survival between groups were assessed with log-rank tests. Multivariable analysis was performed using Coxregression.

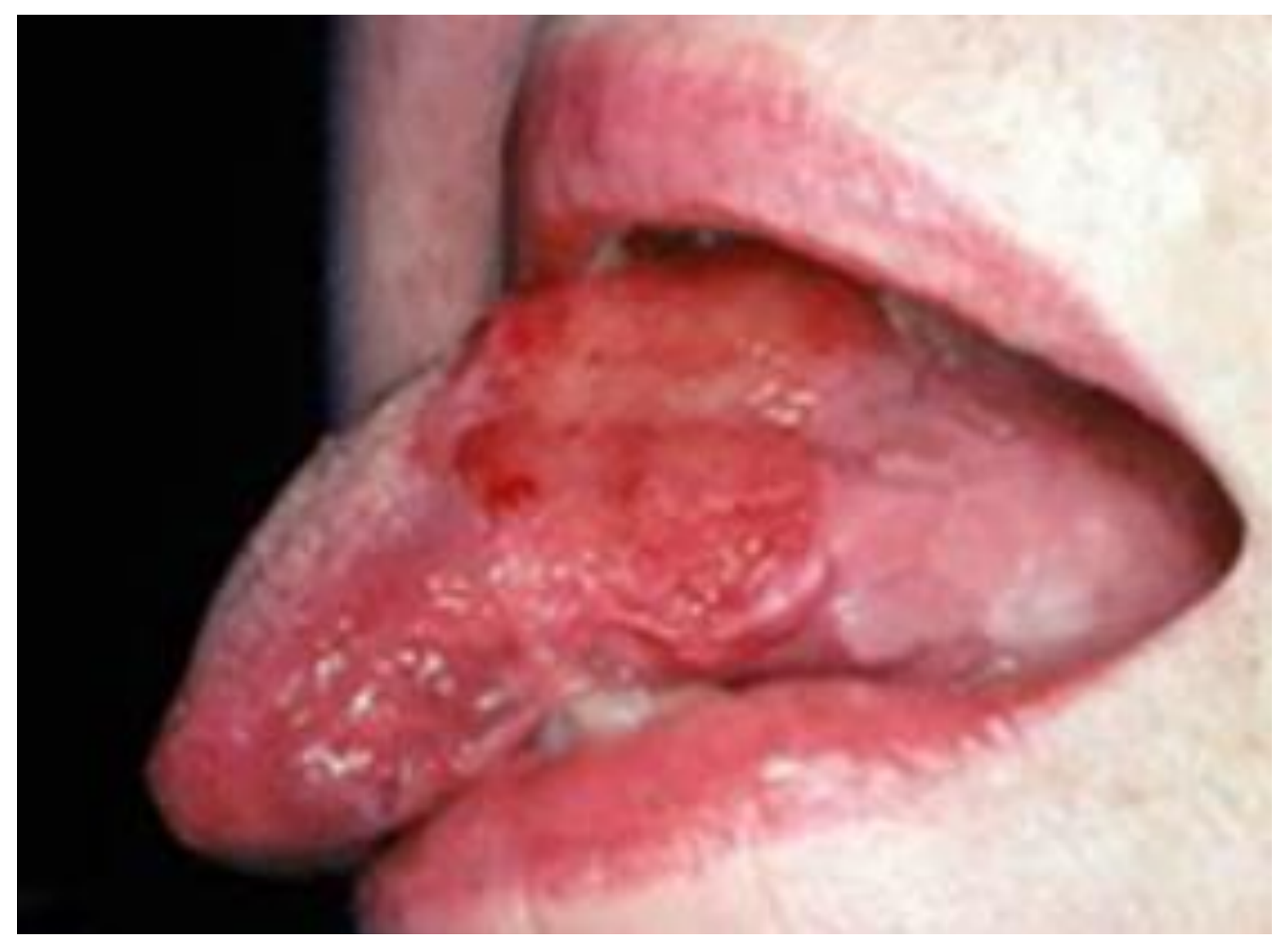

Results: 190 patients were analyzed during the study period. The mean age of patients at presentation was 47.9 years with $77.4 \%$ of patients being male. Buccal mucosa was the most common site $(42.6 \%)$ followed by tongue (32.1\%). More than half of the patients (58.6\%) presented with advanced stage (stage III and IV) disease. 3-year and 5 -year overall survival rates were $71.6 \%$ and $57.9 \%$ respectively with a 5 -year recurrence free survival of $39.5 \%$. Recurrence was seen in 74 patients (41.3\%). Advanced pathological stage (HR: 4.32; 95\%Cl: 1.19-15.61), greater positive lymph node ratio (HR: 2.63; 95\% $\mathrm{Cl}: 1.97-7.07$ ), and incomplete treatment (HR: 1.89; 95\%Cl: 1.01-3.99) were associated with a worse 3 year survival. Patients having recurrence (HR: $1.61 ; 95 \% \mathrm{Cl}$ : 1.022.78) and with greater positive lymph node ratios (HR: 2.39; 95\%Cl: 1.16-4.92) had significantly worse 5 year survival. Kaplan Meier log rank test showed that 3 year and 5 year survival rates were significantly lower ( $p$-value $<0.001$ ) in late stages (III and IV) compared to early stages (I and II).

Conclusion: Factors with significant impact on survival were TNM staging, Greater lymph node ratio and recurrence resulting in a significant decrease in overall survival. Early recognition, comprehensive neck dissections and compliance to complete treatments should be targeted during treatment.

Keywords: Oral cavity squamous cell carcinoma, Prognosis, Neck dissection, Survival. 